UQAM-PHE-95/05

\title{
THE DECAY $H_{2}^{0} \rightarrow g g$ WITHIN THE MINIMAL SUPERSYMMETRIC STANDARD MODEL
}

\author{
HEINZ KÖNIG * \\ Département de Physique \\ Université du Québec à Montréal \\ C.P. 8888, Succ. Centre Ville, Montréal \\ Québec, Canada H3C 3P8
}

\begin{abstract}
I present a detailed SUSY QCD calculation of the decay rate of the lightest Higgs boson $H_{2}^{0}$ into two gluons, where all quarks and scalar quarks are taken within the relevant loop diagrams. I include the mixing of all the scalar partners of the left and right handed quarks and show that their contribution is more than several tens of per cent compared to the quark contribution in the MSSM for some SUSY parameter space. Furthermore the MSSM fermionic contribution is enhanced by several factors for large $\tan \beta$ and large Higgs masses. As a result, the two gluon decay rate of $H_{2}^{0}$ is much larger than the two gluon decay rate of an equal mass standard model Higgs boson. I further compare the decay mode of $H_{2}^{0} \rightarrow g g$ to the similar decay modes of $H_{2}^{0} \rightarrow c \bar{c}$ and $H_{2}^{0} \rightarrow b \bar{b}$ including one loop QCD corrections and show that in some cases $\Gamma\left(H_{2}^{0} \rightarrow g g\right)$ is even higher than $\Gamma\left(H_{2}^{0} \rightarrow c \bar{c}\right)$, although still much smaller than $\Gamma\left(H_{2}^{0} \rightarrow b \bar{b}\right)$.
\end{abstract}

APRIL 1995

* konig@osiris.phy.uqam.ca 


\section{INTRODUCTION}

The Higgs boson is the last particle in the standard model (SM), which yet lacks any experimental evidence. Its discovery therefore is of great importance. The instruments of discovery will be LEP if the Higgs mass is smaller than the Z boson mass and LHC for higher masses. While for a Higgs mass smaller than twice of the gauge boson mass the most important decay modes for its discovery will be $H \rightarrow q \bar{q}$ (here $q=c, b)$ and $H \rightarrow \gamma \gamma$ and to some extent $H \rightarrow g g$, it will be the decay into two W or $\mathrm{Z}$ bosons for higher masses of the Higgs boson.

It is well known that the SM is not a sufficiant model when considering unification theories. The favourite model beyond the SM is its minimal supersymmetric extension (MSSM) [1]. The content of Higgs particles in the MSSM is quite different than the one of the SM: it contains two scalar Higgs bosons $H_{1}^{0}, H_{2}^{0}$, one pseudoscalar $H_{3}^{0}$ and one charged scalar $H^{ \pm}$. The most important point is that the mass of the lightest Higgs particle $m_{H_{2}^{0}}$ has to be smaller than the $\mathrm{Z}$ boson mass at tree level and is enhanced to a maximum value of around $130 \mathrm{GeV}$ when loop corrections are included [2], thus making a SUSY Higgs boson more experimentally reachable.

For a Higgs boson far less massive than the $\mathrm{Z}$ boson, the $H_{2}^{0} \rightarrow \gamma \gamma$ is the most important decay mode and was analyzed in [3] (for the SM) and in [4] (for the MSSM). For values of the Higgs mass up to twice the $\mathrm{W}$ and $\mathrm{Z}$ boson masses the decays $H_{2}^{0} \rightarrow q \bar{q}$ and $H_{2}^{0} \rightarrow g g$ will become more important. The QCD corrections to the first decay mode within the SM were considered in [5] (and references therein) and within the MSSM in [6]. The second decay mode was considered in [7] and two loop QCD corrections within the SM were considered in [8] and found to be relatively large: about $60 \%$. In this paper I show that the MSSM leads to a much higher Higgs into two gluons decay rate than the SM for some supersymmetric parameters, making this decay mode more interesting.

It will be difficult to measure $\Gamma\left(H_{2}^{0} \rightarrow g g\right)$ due to QCD jet background, although it might be experimental measurable at future $e^{+} e^{-}-$colliders [9]. Therefore it is important to consider all kind of models in regards to this decay mode and as I will show the scalar quarks contribution can be several tens of per cent compared to the quark contribution and also to the $H_{2}^{0} \rightarrow c \bar{c}$ decay rate for some SUSY parameter space after summing over all scalar quarks. Furthermore the results presented here can also be used for the Higgs production via gluon fusion in $p \bar{p}$ colliders $[7,8]$.

In the next section I present the calculations and discuss the results in the third section. In the calculation I include the mixing of all scalar partners of the left and right handed quarks, which is expected to be of importance in the top quark sector due to the high top quark mass of $174 \mathrm{GeV}$ reported by the CDF group [10]. In the bottom quark sector I also include one loop effects. As a surprise I also find, that the mixing is not negligible in the charm and strange quark sector independant of the value for $\tan \beta$, the ratio of the Higgs vacuum expectation values (vev's). 


\section{SUSY QCD CORRECTIONS TO $H_{2}^{0} \rightarrow g g$}

In the MSSM there are strong relations among the masses and mixing angles of the Higgs bosons. Given two values e.g. $\tan \beta$ and the light Higgs boson mass $m_{H_{2}^{0}}$ all the other masses and angles can be obtained by the following equations including loop corrections [11]:

$$
\begin{aligned}
\epsilon & =\frac{3 \alpha}{2 \pi} \frac{1}{\sin ^{2} \Theta_{W} \cos ^{2} \Theta_{W}} \frac{1}{\sin ^{2} \beta} \frac{m_{t}^{4}}{m_{Z}^{2}} \ln \left(1+\frac{m_{S}^{2}}{m_{t}^{2}}\right) \\
m_{H_{3}^{0}}^{2} & =\frac{m_{H_{2}^{0}}^{2}\left(m_{Z}^{2}-m_{H_{2}^{0}}^{2}+\epsilon\right)-\epsilon m_{Z}^{2} \cos ^{2} \beta}{m_{Z}^{2} \cos ^{2} 2 \beta-m_{H_{2}^{0}}^{2}+\epsilon \sin ^{2} \beta} \\
m_{H_{1}^{0}}^{2} & =m_{H_{3}^{0}}^{2}+m_{Z}^{2}-m_{H_{2}^{0}}^{2}+\epsilon \\
m_{H^{+}}^{2} & =m_{H_{3}^{0}}^{2}+m_{W}^{2} \\
\tan 2 \alpha & =\tan 2 \beta \frac{m_{H_{3}^{0}}^{2}+m_{Z}^{2}}{m_{H_{3}^{0}}^{2}-m_{Z}^{2}+\epsilon / \cos 2 \beta} \quad-\frac{\pi}{2} \leq \alpha \leq 0
\end{aligned}
$$

Here $m_{S}$ is the soft supersymmetry breaking scalar mass. In eq.(1) I have neglected the contribution coming from the bilinear Higgs mass term $\mu$ and $A_{f}$ the parameters descriping the strengths of nonsupersymmetric trilinear scalar interactions. Their inclusion only changes the results by a few $\mathrm{GeV}$ as stated in [11].

In the SM the decay mode of the Higgs boson into two gluons occur via one loop diagrams with all quarks taken within the loop as shown in Fig.1. After summation over both directions of the interior fermions momenta or, which is topological equivalent, adding the diagram where $p_{1} \leftrightarrow p_{2}$ the amplitude is finite and the result is given by:

$$
\begin{aligned}
i M_{q} & =+g_{2} \frac{g_{s}^{2}}{(4 \pi)^{2}} \frac{m_{Z}^{2}}{m_{W}} \frac{4}{q^{2}}\left(p_{1} p_{2} g_{\alpha \beta}-p_{1 \beta} p_{2 \alpha}\right) \epsilon_{p_{1}}^{* \alpha} \epsilon_{p_{2}}^{* \beta} T_{q} \\
T_{q} & =\sum_{q} \frac{m_{q}^{2}}{m_{Z}^{2}} K^{q H_{2}^{0}}\left[2-\left(1-4 \lambda_{q}\right) I_{q}\right]
\end{aligned}
$$

with $q^{2}=m_{H_{2}^{0}}^{2}$ on mass shell and $K^{u H_{2}^{0}}=\cos \alpha / \sin \beta$ and $K^{d H_{2}^{0}}=-\sin \alpha / \cos \beta$ and the function $I_{q}$ defined by

$$
\begin{aligned}
I_{q} & =\left\{\begin{array}{cc}
-2\left[\arcsin \left(\frac{1}{2 \sqrt{\lambda_{q}}}\right)\right]^{2}, & 1 / 4 \leq \lambda_{q} \\
{\left[\ln \left(\frac{r_{+}}{r_{-}}\right)^{2}-\pi^{2}\right] / 2+i \pi \ln \left(\frac{r_{+}}{r_{-}}\right),} & \lambda_{q}<1 / 4
\end{array}\right. \\
r_{ \pm} & =1 \pm\left(1-4 \lambda_{q}\right)^{\frac{1}{2}}
\end{aligned}
$$

with $\lambda_{q}=\left(m_{q} / m_{H_{2}^{0}}\right)^{2}$. Before I present the results of the scalar quarks contribution to the Higgs decay into two gluons I first want to comment on their mass matrices in the MSSM. The mixing term of the scalar partners of the left and right handed 
quarks is proportional to the quark masses and hence was neglected before the top quark was discovered as very heavy. In the calculation here I include the mixing of all scalar quarks and present the result in their mass eigenstates, that is instead of the current eigenstates $\tilde{q}_{L, R}$ I work with the mass eigenstates

$$
\tilde{q}_{1}=\cos \Theta \tilde{q}_{L}+\sin \Theta \tilde{q}_{R} \quad \tilde{q}_{2}=-\sin \Theta \tilde{q}_{L}+\cos \Theta \tilde{q}_{R}
$$

In the scalar up quark sector we have the following matrix [12]:

$$
M_{\tilde{u}}^{2}=\left(\begin{array}{cc}
m_{\tilde{u}_{L}}^{2}+m_{u}^{2}+0.35 D_{Z} & -m_{u}\left(A_{u}+\mu \cot \beta\right) \\
-m_{u}\left(A_{u}+\mu \cot \beta\right) & m_{\tilde{u}_{R}}^{2}+m_{u}^{2}+0.15 D_{Z}
\end{array}\right)
$$

Here $D_{Z}=m_{Z}^{2} \cos 2 \beta, 0.35=T_{3}^{u}-e_{u} \sin \Theta_{W}$ and $0.15=e_{u} \sin \Theta_{W} \cdot m_{\tilde{u}_{L, R}}$ are soft SUSY breaking mass terms, $A_{u}$ the parameter from the trilinear scalar interaction and $\mu$ the mixing mass term of the Higgs bosons. Here $u$ stands for all three families up, charm and top.

For the mass matrix of the bottom quark, we have to be more careful since it is well known that charged Higgsino exchange in left handed scalar down quark self mass diagrams leads to a scalar down quark squared mass with a term proportional to the up quark mass squared and to flavour changing gluino-scalar down quark-down quark couplings [13]. For the mass matrix of the scalar down quark we therefore have to take at 1 loop level:

$$
M_{\tilde{d}}^{2}=\left(\begin{array}{cc}
m_{\tilde{d}_{L}}^{2}+m_{d}^{2}-0.42 D_{Z}-|c| m_{u}^{2} & -m_{d}\left(A_{d}+\mu \tan \beta\right) \\
-m_{d}\left(A_{d}+\mu \tan \beta\right) & m_{\tilde{d}_{R}}^{2}+m_{d}^{2}-0.08 D_{Z}
\end{array}\right)
$$

with $T_{3}^{d}-e_{d} \sin \Theta_{W}=-0.42$ and $e_{d} \sin \Theta_{W}=-0.08$. Here $d$ stands for all three families down, strange and bottom. The value of $c$ is negative and of order $1(|c|$ increases with the soft SUSY breaking mass term $m_{S}$ and decreases with the top quark mass [14]). In the following I take $c=-1$, although I keep in mind that it is more likely smaller. In the calculation it turns out that the mixing of the first generation is negligible as expected, whereas in the second generation $\sin \Theta_{q} \simeq$ $0.1-0.5$ (the last value only for $\tan \beta \gg 1$ ) and therefore not negligible. In the third generation $\sin \Theta_{q} \simeq 1 / \sqrt{2}$ due to the heavy top quark mass. For the scalar bottom quark the mixing angle only becomes that big when $\tan \beta \gg 1$.

In the MSSM we have to add up the two diagrams shown in Fig.2. After summation the amplitude is finite and the result is given by:

$$
\begin{aligned}
i M_{\tilde{q}}= & -\frac{g_{2}}{\cos \Theta_{W}} \frac{g_{s}^{2}}{(4 \pi)^{2}} \frac{4 m_{Z}}{q^{2}}\left(p_{1} p_{2} g_{\alpha \beta}-p_{1 \alpha} p_{2 \alpha}\right) \epsilon_{p_{1}}^{* \alpha} \epsilon_{p_{2}}^{* \beta} T_{\tilde{q}} \\
T_{\tilde{q}}= & \sum_{q}\left\{\left[\cos ^{2} \Theta_{q} K_{11}^{\tilde{q} H_{2}^{0}}+\sin ^{2} \Theta_{q} K_{22}^{\tilde{q} H_{2}^{0}}+2 \sin \Theta_{q} \cos \Theta_{q} K_{12}^{\tilde{q} H_{2}^{0}}\right]\left(1+2 \lambda_{\tilde{q}_{1}} I_{\tilde{q}_{1}}\right)\right. \\
& \left.+\left[\sin ^{2} \Theta_{q} K_{11}^{\tilde{q} H_{2}^{0}}+\cos ^{2} \Theta_{q} K_{22}^{\tilde{q} H_{2}^{0}}-2 \sin \Theta_{q} \cos \Theta_{q} K_{12}^{\tilde{q} H_{2}^{0}}\right]\left(1+2 \lambda_{\tilde{q}_{2}} I_{\tilde{q}_{2}}\right)\right\}
\end{aligned}
$$




$$
\begin{aligned}
& K_{11}^{\tilde{u} H_{2}^{0}}=-\left(\frac{1}{2}-e_{u} s_{W}^{2}\right) \sin (\alpha+\beta)+\left(\frac{m_{u}}{m_{Z}}\right)^{2} \frac{\cos \alpha}{\sin \beta} \\
& K_{22}^{\tilde{u} H_{2}^{0}}=-e_{u} s_{W}^{2} \sin (\alpha+\beta)+\left(\frac{m_{u}}{m_{Z}}\right)^{2} \frac{\cos \alpha}{\sin \beta} \\
& K_{12}^{\tilde{u} H_{2}^{0}}=K_{21}^{\tilde{u} H_{2}^{0}}=\frac{1}{2} \frac{m_{u}}{m_{Z}^{2}} \frac{1}{\sin \beta}\left(A_{u} \cos \alpha+\mu \sin \alpha\right) \\
& K_{11}^{\tilde{d} H_{2}^{0}}=\left(\frac{1}{2}+e_{d} s_{W}^{2}\right) \sin (\alpha+\beta)-\left(\frac{m_{d}}{m_{Z}}\right)^{2} \frac{\sin \alpha}{\cos \beta} \\
& K_{22}^{\tilde{d} H_{2}^{0}}=-e_{d}^{2} s_{W}^{2} \sin (\alpha+\beta)-\left(\frac{m_{d}}{m_{Z}}\right)^{2} \frac{\sin \alpha}{\cos \beta} \\
& K_{12}^{\tilde{d} H_{2}^{0}}=K_{21}^{\tilde{d} H_{2}^{0}}=-\frac{1}{2} \frac{m_{d}}{m_{Z}^{2}} \frac{1}{\cos \beta}\left(A_{d} \sin \alpha+\mu \cos \alpha\right)
\end{aligned}
$$

with $s_{W}^{2}=\sin ^{2} \Theta_{W}$ and again $q^{2}=m_{H_{2}^{0}}^{2}$ on mass shell. Note that the non diagonal terms $K_{12}^{\tilde{q} H_{2}^{0}}$ in $T_{\tilde{q}}$ only contribute when the scalar mass eigenstates differ, which mainly is the case for the third generation. The amplitudes in eq.(2) and eq.(7) lead to the following decay rate:

$$
\Gamma\left(H_{2}^{0} \rightarrow g g\right)=\frac{\alpha \alpha_{s}^{2}}{8 \pi^{2} \sin ^{2} \Theta_{W} \cos ^{2} \Theta_{W}} \frac{m_{Z}^{2}}{m_{H_{2}^{0}}}\left|T_{q}-T_{\tilde{q}}\right|^{2}
$$

Here I take the strong coupling constant Higgs mass dependant as given in eq.(9) of Ref. [5]. If $T_{\tilde{q}}$ is set to 0 eq.(8) reproduce eq.(2.29) given in [15]. Before I discuss the results I want to make some comments. The amplitudes given in eq.(2) and eq.(7) can also be used when considering Higgs boson production via gluon fusion. However there one also has to include the $\mathrm{Z}$ boson production via gluon fusion. This becomes important when considering heavy lepton production at hadron colliders $[16,17]$. It is also interesting to see that it is possible to produce $\mathrm{Z}$ bosons via gluon fusion, whereas the $\mathrm{Z}$ decay into two gluons is identical to 0 when the $\mathrm{Z}$ boson is on mass shell $q^{2}=m_{Z}^{2}$, although its amplitude is not (Yang's theorem [18]).

The scalar quarks do not contribute to the $\mathrm{Z}$ boson decay into two gluons (after adding the diagram with $p_{1} \leftrightarrow p_{2}$ the result is identical to 0 after Feynman integration). When quarks are taken within the loop only the terms with $\gamma_{5}$ survive after integration, which also indicates that because of charge conservation no photons are produced by gluon fusion (Furry's theorem). The amplitude is then given by:

$$
i M_{q Z}=\frac{g_{2}}{\cos \Theta_{W}} \frac{g_{s}^{2}}{(4 \pi)^{2}}\left(c_{R}^{q Z}-c_{L}^{q Z}\right) \frac{4}{q^{2}}\left\{1+2 \lambda_{q} I_{q}\right\} i \epsilon_{\alpha \beta \delta \gamma} p_{1}^{\delta} p_{2}^{\gamma} q_{\mu} \epsilon_{p_{1}}^{* \alpha} \epsilon_{p_{2}}^{* \beta} \epsilon_{q}^{\mu}
$$

with $c_{L}^{q Z}=T_{3 q}-e_{q} s_{W}^{2}$ and $c_{R}^{q Z}=-e_{q} s_{W}^{2}$. Eq.(9) agrees with eq.(1) in [19]. Eq.(2), eq.(7) and eq.(9) reproduce the results presented in [17]. When considering the Z decay I obtain after squaring and summing over the polarization states of the $\mathrm{Z}$ 
boson a term proportional to $q_{\mu} q_{\nu}\left(g^{\mu \nu}-q^{\mu} q^{\nu} / m_{Z}^{2}\right) \equiv 0$ on mass shell $q^{2}=m_{Z}^{2}$, whereas when the $\mathrm{Z}$ boson is produced via gluon fusion and then be treated as a propagator I have a term proportional to $q_{\mu}\left(g^{\mu \nu}-q^{\mu} q^{\nu} / m_{Z}^{2}\right) /\left(q^{2}-m_{Z}^{2}\right) \equiv-q^{\nu} / m_{Z}^{2}$ and therefore of importance in the gluon fusion process.

In the next section I will discuss the results of the lightest supersymmetric Higgs boson into two gluons decay rate obtained in eq.(8).

\section{DISCUSSIONS}

As input parameters I take $m_{\text {top }}=174 \mathrm{GeV}$ and for the strong coupling constant the Higgs mass dependant function $\alpha_{s}=12 \pi /\left[(33-2 n) \ln \left(m_{H_{2}^{0}}^{2} / \Lambda_{\mathrm{QCD}}^{2}\right)\right]$ with $n=5$ and $\Lambda_{\mathrm{QCD}}=180 \mathrm{MeV}$. I use $m_{\tilde{u}_{L}}=m_{\tilde{u}_{R}}=m_{\tilde{d}_{L}}=m_{\tilde{d}_{R}}=m_{S}=A_{\tilde{u}}=A_{\tilde{d}}$. To see how big the contribution of the scalar quarks compared to the quarks is I plot in Fig. 3 the ratio $\Gamma^{\tilde{q}+q} / \Gamma^{q}$ of the decay rate $\Gamma\left(H_{2}^{0} \rightarrow g g\right)$ as function of the soft SUSY breaking scalar mass $m_{S}$ for a fixed value of $\mu=250 \mathrm{GeV}$ and two different values of the Higgs mass $m_{H_{2}^{0}}=60 \mathrm{GeV}$ and $120 \mathrm{GeV}$ and 3 different values of $\tan \beta=3$ (solid line), 10 (dashed line) and 60 (dotted line). Higher values of $\tan \beta$ are preferred in superstring inspired $E_{6}$ and $S O(10)$ models. For $\Gamma^{q}$ and $\Gamma^{\tilde{q}+q}$ I have taken $T_{q}$ as given in eq.(2) with the couplings $K^{q H_{2}^{0}}$, that is including the large enhancements (relative to the $\mathrm{SM}$ ) due to large $\tan \beta$. As a result I have that for small values of $m_{S}$ the scalar quarks contribute even more than the quarks, although their contribution decrease rapidly and remains only a few per cent for $m_{S}>600 \mathrm{GeV}$. For $\tan \beta=60$ the scalar quarks contribution diminishes the ratio for $m_{S}<350$ $\mathrm{GeV}$ and enhances it for higher values. The influence of $\mu$ is very small for small $\tan \beta$ and becomes more important for very high $\tan \beta$ values. For small values of $\tan \beta$ higher values of $\mu$ enhance the decay rate a little bit. For high values of $\tan \beta$ it is the other way around and the differences are larger. A negative value for $\mu$ leads to a bit smaller values of the decay rate.

In Fig.4 I have plotted the ratio of the Higgs into two gluons decay rate of the MSSM compared to the SM, that is $\Gamma^{\tilde{q}+q} / \Gamma^{q}$, where I have taken $\Gamma^{q}$ as it is in the SM that is without the couplings $K^{q H_{2}^{0}}$, whereas I included them in $\Gamma^{\tilde{q}+q}$. For the Higgs mass I have taken $60 \mathrm{GeV}$. As a result I have that for scalar masses smaller than $500 \mathrm{GeV}$ the Higgs into two gluons decay rate is enhanced by several tens of per cents in the MSSM and gives the same result than the SM for higher values of the scalar masses. As in Fig.3 for $\tan \beta=60$ and $m_{S}<350 \mathrm{GeV}$ the decay is diminished.

In Fig.5 I have done the same as in Fig.4 but for a Higgs mass of $m_{H_{2}^{0}}=120$ $\mathrm{GeV}$. Here the results are quite different than in Fig.4. As a result I have that in the MSSM the Higgs into two gluon decay rate is enhanced by several tens of per cent for $\tan \beta=3$, by a factor of $2-3$ for $\tan \beta=10$ and $m_{S}<300 \mathrm{GeV}$ and even by an order of magnitude for $\tan \beta=60$ with the highest contribution for a scalar mass around $550 \mathrm{GeV}$.

As I have shown in Fig.3 the scalar quarks decouples for $m_{S}>600 \mathrm{GeV}$. The reason why the branching ratio as shown in Fig. 5 is still larger than 1 even for higher 
values of the scalar mass is that $\Gamma^{q}$ is quite different in the MSSM with the couplings $K^{q H_{2}^{0}}$ than it is in the SM without these couplings. In the SM the main contribution is from the heavy top quark and a few per cent from the bottom quark. The contribution of the other quarks are negligible due to their small masses. In the MSSM the bottom quark contribution becomes as important as the top quark contribution for large $\tan \beta$ values eg. the ratio $\Gamma_{\mathrm{SM}}^{q} / \Gamma_{\mathrm{MSSM}}^{q}$ becomes very small depending on the size and $\operatorname{sign}$ of $\sin \alpha$, which becomes relative large around \pm 0.5 and thus leading to large values of $\Gamma^{\tilde{q}+q} / \Gamma^{q}$ as seen in Fig.5. For a small Higgs mass of $60 \mathrm{GeV}$ as I have taken in Fig. $4 \sin \alpha$ remains always smaller than around $-2 \times 10^{-2}$ and therefore keeps the bottom quark mass contribution as small as in the SM.

Some curves in Fig.4-5 start at different values of $m_{S}$ because, for values of $m_{S}$ higher than $600 \mathrm{GeV}$ I obtain an unphysical negative mass squared for the pseudo particle $H_{3}^{0}$ if $\tan \beta=3$; for $\tan \beta=10$ the unphysical region is when $m_{S} \simeq 650$ $\mathrm{GeV}$; whereas for $\tan \beta=60 m_{H_{3}^{0}}$ is physical for all $m_{S}$.

A negative eigenvalue of the scalar bottom quark mass also occurs if $m_{S}<200$ $\mathrm{GeV}$ for $\tan \beta=3$ and 10 or $m_{S}<300 \mathrm{GeV}$ for $\tan \beta=60$. Here the parameter $c$ in the scalar bottom mass squared matrix eq.(6) is of importance, neglecting it would allow us to use $m_{S}$ as small as $100 \mathrm{GeV}$ (for $\tan \beta=3$ ) without running into one negative mass eigenvalue of the scalar bottom quark mass, with the result that $\Gamma^{\tilde{q}+q}$ can become much larger than $\Gamma^{q}$. Unfortunately even for smaller values for $c \simeq-0.5$ I obtain negative values with such a small scalar mass. Since $c$ cannot be neglected when including loop corrections I excluded those regions in the figures.

In Fig.4 and Fig.5 I also have plotted the ratio of the decay rates $\Gamma\left(H_{2}^{0} \rightarrow\right.$ $g g) / \Gamma\left(H_{2}^{0} \rightarrow c \bar{c}\right)$. For the decay rate $\Gamma\left(H_{2}^{0} \rightarrow b \bar{b}, c \bar{c}\right)$ I used the tree result including the SM QCD corrections as given in eq.(8) of Ref. [5] with the changes of the tree level couplings within the MSSM. I did not include the SUSY QCD correction, because they are far smaller than the SM QCD correction as I have shown in [6]. There I showed that for $\tan \beta=1$ SUSY QCD corrections do not contribute at all to this decay mode $(\sin (\alpha+\beta)=0)$ and presented the results in the limit of $\tan \beta \gg 1$. There I did not include the mixing of the scalar charm and bottom quarks, but since $m_{\tilde{c}_{1}} \approx m_{\tilde{c}_{2}}$ even a large mixing angle will not change the results for the decay rate $\Gamma\left(H_{2}^{0} \rightarrow c \bar{c}\right)$ presented there. This might not be true for $\Gamma\left(H_{2}^{0} \rightarrow b \bar{b}\right)$ which I did not consider here since it is much higher than $\Gamma\left(H_{2}^{0} \rightarrow g g\right)$, by a factor of at least 50. Therefore in Fig.4 and Fig.5 I only compared the Higgs into two gluons decay rate with the one to the charm- anti-charm quarks. In Fig.4, the dependence of the ratio to the scalar mass $m_{S}$ is very small, since $\Gamma^{\tilde{q}+q}$ becomes very small and the dependence of $\Gamma^{c \bar{c}}$ to $m_{S}$ is only via the angles $\cos ^{2} \alpha / \sin ^{2} \beta$, which is compensated by the $K^{t H_{2}^{0}}$ coupling in the $T_{q}$ term. For a very large scalar mass the ratio remains constant with a value of around 0.31 independant of $\tan \beta$. A quite different result I obtain in Fig.5, especially again for $\tan \beta=60$, for the same reason as explained above. The shape of the figures is quite similar compared to the ratio $\Gamma^{\tilde{q}+q} / \Gamma^{q}$. For scalar masses much higher than $1 \mathrm{TeV}$ the ratio remains constant with a value of around 1 independant of $\tan \beta$.

Finally in Fig.6 I show the influence of the Higgs mass to the decay rate 
$\Gamma\left(H_{2}^{0} \rightarrow g g\right)$ for a fixed value of $\mu=250 \mathrm{GeV}$ and $m_{S}=300 \mathrm{GeV}$ and three different values of $\tan \beta=3,10$ and 60 . In the case $\tan \beta=3$ I obtain negative values for the mass squared of the pseudo Higgs $H_{3}^{0}$ in the range of $95<m_{H_{2}^{0}}<105$ $\mathrm{GeV}$ which therefore has to be excluded. As a result I have that $\Gamma^{\tilde{q}+q} / \Gamma^{q}$ is weakly dependant of the Higgs mass, not so $\Gamma^{\tilde{q}+q} / \Gamma^{c \bar{c}}$, which shape is basically dominated by $\cos \alpha$ and $\sin \beta$.

\section{CONCLUSION}

In this paper I presented the corrections to the lightest MSSM Higgs boson decay into two gluons when scalar quarks are taken within the loop. I included in my calculation the mixing of all scalar quarks although it only becomes important for the second and third generation. I have shown that scalar quarks lead to a decay rate of the same order as the quarks in the SM for values of $m_{S}$ smaller than 600 $\mathrm{GeV}$. In the SM the largest contribution comes from the top quark due to the $m_{q}^{2}$ in $T_{q}$. In the MSSM the $T_{\tilde{q}}$ are of the same order for all scalar quarks and therefore contribute many more terms to $\Gamma\left(H_{2}^{0} \rightarrow g g\right)$ than the SM alone. Furthermore in the MSSM the $T_{q}$ can become much larger than in the SM for $\tan \beta \gg 1$ and large negative or positive $\sin \alpha$. I also have shown that the Higgs into two gluon decay rate can become even larger than the decay into charm- anti-charm quarks for $\tan \beta=3$ and the Higgs mass larger than around $80 \mathrm{GeV}$ and for $\tan \beta=10$ and 60 and the Higgs mass larger than the $\mathrm{Z}$ boson mass, but still remains more than a factor of 50 smaller than its decay into bottom- anti-bottom quarks.

Although the decay of the Higgs into two gluons will be difficult to measure it is of importance to know how big the influence of models beyond the SM might be. Furthermore it might be measurable at future $e^{+} e^{-}-$colliders [9]. The amplitudes given here can also be used when considering Higgs and Z boson production in hadron colliders via gluon fusion.

\section{ACKNOWLEDGMENTS}

The author would like to thank the physics department of Carleton university for the use of their computer facilities. The author would also like to thank M.A. Doncheski, for carefully reading this manuscript and usefull discussions and also A. Djouadi for drawing my attention after submission of this paper to reference [20] about a similiar subject, which pointed out a mistake in eq.(7). The figures were done with the very user friendly program PLOTDATA from TRIUMF.

This work was partially funded by funds from the N.S.E.R.C. of Canada and les Fonds F.C.A.R. du Québec.

\section{REFERENCES}

[ 1] H.E. Haber and G.L. Kane, Phys.Rep.117(1985)75.

[ 2] H. Haber and R. Hempfling, Phys. Rev. Lett. 66 (1991)1815; Y. Okada et al., Prog. Theor. Phys. 85(1991)1; Phys. Lett. 262B(1991)54; J. Ellis et al., ibid 
257B (1991)83; for a more general analysis leading to a higher mass limit of about $150 \mathrm{GeV}$ see G.L. Kane et al., Phys. Rev. Lett. 70(1993)2686.

[ 3] L. Resnick, M.K. Sundaresan and P.J. Watson, Phys. Rev. D8(1973)172; A.J. Vainstein et al., Yad. Fiz. 30(1979)1368 [Sov.J.Nucl.Phys.30(1979)711].

[4] R. Bates, J.N. Ng and P. Kalyniak, Phys. Rev. D34(1986)172.

[5] P. Kalyniak et al., Phys. Rev. D43(1991)3664.

[ 6] H. König, Phys. Rev. D47(1993)4995.

[7] F. Wilczek, Phys. Rev. Lett.39(1977)1304; H.M. Georgi et al., Phys. Rev. Lett. 40(1978)692; J. Ellis et al., Phys. Lett.83B(1979)339; T. Rizzo, Phys. Rev. D22(1980)178.

[ 8] A. Djouadi, M. Spira and P.M. Zerwas, Phys. Lett. 264B(1991)440; M. Spira et al., preprint DESY 94-123, CERN-TH/95-30, GPP-UdeM-TH-95-16 (1995), hep-ph/9504378.

[ 9] Proceedings of the Workshop " $e^{+} e^{-}$Collisions at $500 \mathrm{GeV}$ : The Physics Potential", DESY Report 92-123A; August 1992, P. Zerwas, ed.

[10] The CDF collaboration (F. Abe et al.), Phys. Rev. Lett. 73(1994)225.

[11] A. Djouadi, Int.J.Mod.Phys.A10(1995)1

[12] A. Djouadi, M. Drees and H. König, Phys.Rev.D48(1993)3081.

[13] J. Ellis and D.V. Nanopoulos, Phys. Lett. 110B(1982)44;R. Barbieri and R. Gato, Phys. Lett. 110B(1982)210;M.J. Duncan, Nucl.Phys.B221(1983)285; J.F. Donughue, H.P. Nilles and D. Wyler, Phys. Lett. 128B(1983)55;E. Franco and M. Mangano, Phys.Lett. 135B(1984)445;A. Bouquet, J. Kaplan and C.A. Savoy, Phys. Lett.148B(1984)69.

[14] S. Bertolini, F. Borzumati and A. Masiero, Phys. Lett194B(1987)551. Erratum: ibid198B(1987)590.

[15] J.F. Gunion and et al., The Higgs Hunter's Guide, (Addison-Wesley, Redwood City, CA, 1990.

[16] M. Boyce, M.A. Doncheski and H. König, work in progress.

[17] J.E. Cieza Montalvo, O.J.P.Éboli and S.F. Novaes, Phys.Rev.D46(1992)181.

[18] C.N. Yang, Phys.Rev. 77(1950)242.

[19] D.A. Dicus and P. Roy, Phys.RevD44(1991)1593.

[20] B. Kileng, Z.Phys.C.-Part.and Fields 63 (1994)87.

\section{FIGURE CAPTIONS}

Fig.1 The penguin diagram with up and down quarks within the loop leading to the $H_{2}^{0} \rightarrow g g$ decay.

Fig.2 The penguin diagrams with scalar up and scalar down quarks within the loop leading to the $H_{2}^{0} \rightarrow g g$ decay.

Fig.3 The ratio $\Gamma^{\tilde{q}+q} / \Gamma^{q}$ of the decay $H_{2}^{0} \rightarrow g g$ as a function of the soft SUSY breaking scalar mass term $m_{S}$ for a fixed value of $\mu=250 \mathrm{GeV}$ and $m_{H_{2}^{0}}=60 \mathrm{GeV}$ and $120 \mathrm{GeV}$ and three different values of $\tan \beta=3$ (solid line), 10 (dashed line) and 60 (dotted line). For $\tan \beta=3$ the upper curve is for $m_{H_{2}^{0}}=120 \mathrm{GeV}$ and the lower curve for $60 \mathrm{GeV}$. For $\tan \beta=10$ and 60 it is the other way around. Here 
I have taken for $\Gamma^{q}$ the equation as it is given in eq.(2) including the couplings $K^{q H_{2}^{0}}$.

Fig.4 The ratio $\Gamma^{\tilde{q}+q} / \Gamma^{q}$ and $\Gamma^{\tilde{q}+q} / \Gamma^{c \bar{c}}$ of the decay $H_{2}^{0} \rightarrow g g$ as a function of the soft SUSY breaking scalar mass term $m_{S}$ for a fixed value of $\mu=250 \mathrm{GeV}$ and $m_{H_{2}^{0}}=60 \mathrm{GeV} \cdot \tan \beta$ as in Fig.3. Here I have taken for $\Gamma^{q}$ the equation as it is given in the SM, that is without the couplings $K^{q H_{2}^{0}}$.

Fig.5 The same as Fig.4 with $m_{H_{2}^{0}}=120 \mathrm{GeV}$. The upper curves are for $\Gamma^{\tilde{q}+q} / \Gamma^{c \bar{c}}$. For $\tan \beta=3$ and $m_{S} \geq 600 \mathrm{GeV}$ the pseudo Higgs becomes a negative mass squared. For $\tan \beta=10$ the same happens for a small region when $m_{S} \approx 650$ $\mathrm{GeV}$.

Fig.6 The same as Fig.4 but as a function of of $m_{H_{2}^{0}}$ for fixed values $m_{S}=300 \mathrm{GeV}$ and $\mu=250 \mathrm{GeV}$ and $\tan \beta$ as in Fig.3. Here the upper curves at the higher Higgs masses are for $\Gamma^{\tilde{q}+q} / \Gamma^{c \bar{c}}$. In the case $\tan \beta=3$ the pseudo scalar mass squared $m_{H_{3}^{0}}^{2}$ becomes negative in the range $95<m_{H_{2}^{0}}<105 \mathrm{GeV}$. 
This figure "fig1-1.png" is available in "png" format from: http://arxiv.org/ps/hep-ph/9504433v2 
This figure "fig2-1.png" is available in "png" format from: http://arxiv.org/ps/hep-ph/9504433v2 
This figure "fig1-2.png" is available in "png" format from: http://arxiv.org/ps/hep-ph/9504433v2 
This figure "fig2-2.png" is available in "png" format from: http://arxiv.org/ps/hep-ph/9504433v2 
This figure "fig1-3.png" is available in "png" format from: http://arxiv.org/ps/hep-ph/9504433v2 